\title{
1 A Newcastle disease virus-vector expressing a prefusion-stabilized spike protein of SARS-CoV-2 2 induces protective immune responses against prototype virus and variants of concern in mice and 3 hamsters \\ 5 Weina Sun ${ }^{1}$, Yonghong Liu ${ }^{1}$, Fatima Amanat ${ }^{1,2}$, Irene González-Domínguez ${ }^{1}$, Stephen McCroskery ${ }^{1,3}$, \\ 6 Stefan Slamanig ${ }^{1}$, Lynda Coughlan ${ }^{6,12}$, Victoria Rosado ${ }^{1}$, Nicholas Lemus ${ }^{1}$, Sonia Jangra ${ }^{1,4}$, Raveen \\ 7 Rathnasinghe ${ }^{1,2,4}$, Michael Schotsaert ${ }^{1,4}$, Jose Martinez ${ }^{1}$, Kaori Sano ${ }^{1}$, Ignacio Mena ${ }^{1,4}$, Bruce L Innis ${ }^{10}$, \\ 8 Ponthip Wirachwong ${ }^{8}$, Duong Huu Thai ${ }^{7}$, Ricardo Das Neves Oliveira ${ }^{9}$, Rami Scharf ${ }^{10}$, Richard Hjorth ${ }^{10}$, \\ 9 Rama Raghunandan ${ }^{10}$, Florian Krammer ${ }^{1,11}$, Adolfo García-Sastre ${ }^{1,3,4,5,11}$ and Peter Palese $\mathrm{K}^{1,3^{*}}$
}

${ }^{1}$ Department of Microbiology, Icahn School of Medicine at Mount Sinai, New York, NY 10029, USA

${ }^{2}$ Graduate School of Biomedical Sciences, Icahn School of Medicine at Mount Sinai, New York, NY 10029, USA

${ }^{3}$ Department of Medicine, Icahn School of Medicine at Mount Sinai, New York, NY 10029, USA

${ }^{4}$ Global Health Emerging Pathogens Institute, Icahn School of Medicine at Mount Sinai, New York, NY 10029, USA

${ }^{5}$ The Tisch Cancer Institute, Icahn School of Medicine at Mount Sinai, New York, NY 10029, USA

${ }^{6}$ University of Maryland School of Medicine, Department of Microbiology and Immunology, Baltimore, MD 21201, USA

${ }^{7}$ Institute of Vaccines and Medical Biologicals, Nha Trang City, Khanh Hoa Province, Vietnam

${ }^{8}$ The Government Pharmaceutical Organization, Bangkok 10400, Thailand

${ }^{9}$ Instituto Butantan, São Paulo, SP, 05503-900, Brazil

${ }^{10}$ PATH, Center for Vaccine Access and Innovation, Washington, DC 20001, USA

${ }^{11}$ Department of Pathology, Icahn School of Medicine at Mount Sinai, New York, NY 10029, USA

${ }^{12}$ University of Maryland School of Medicine, Center for Vaccine Development and Global Health (CVD), Baltimore, MD 21201, USA

* To whom correspondence should be addressed: peter.palese@mssm.edu

\section{Abstract}

Rapid development of coronavirus disease 2019 (COVID-19) vaccines and expedited authorization

32 for use and approval has been proven beneficial to mitigate severe acute respiratory syndrome coronavirus

332 (SARS-CoV-2) spread and given hope in this desperate situation. It is believed that sufficient supplies 34 and equitable allocations of vaccines are necessary to limit the global impact of the COVID-19 pandemic 
and the emergence of additional variants of concern. We have developed a COVID-19 vaccine based on Newcastle disease virus (NDV) that can be manufactured at high yields in embryonated eggs. Here we provide evidence that the NDV vector expressing an optimized spike antigen (NDV-HXP-S), upgraded from our previous construct, is a versatile vaccine that can be used live or inactivated to induce strong antibody responses and to also cross-neutralize variants of concern. The immunity conferred by NDV-HXP$\mathrm{S}$ effectively counteracts SARS-CoV-2 infection in mice and hamsters. It is noteworthy that vaccine lots produced by existing egg-based influenza virus vaccine manufacturers in Vietnam, Thailand and Brazil exhibited excellent immunogenicity and efficacy in hamsters, demonstrating that NDV-HXP-S vaccines can be quickly produced at large-scale to meet global demands.

\section{Introduction}

The coronavirus disease 2019 (COVID-19) pandemic caused by severe acute respiratory syndrome coronavirus 2 (SARS-CoV-2) has brought disastrous outcomes to public health, education and economics worldwide. Emerged variants of concern that are currently circulating could threaten the prior preventive achievements if not managed properly. The rollout of COVID-19 vaccines such as mRNA vaccines (Pfizer and Moderna), inactivated virus vaccines (Sinovac, Sinopharm), adenovirus-vector vaccines (AstraZeneca, CanSino Biologics, Gamaleya Research Institute and J\&J) have helped to contain the spread of the virus tremendously, stressing the importance of prophylactic measures. However, despite the high efficacy of mRNA vaccines, availability of such vaccines to developing countries is restricted due to cold or ultra-cold chain requirements and lack of manufacturing infrastructure and capacity. Indeed, with North America and Europe having the highest vaccination rates, vaccine resources are much less accessible to developing countries in Latin America, Asia and Africa (1). Such inequitable availability of vaccine delays prompt control of COVID-19 and increases the risk of additional variants to emerge. This highlights the urgent need for affordable vaccines that can be produced locally.

We have previously developed a Newcastle disease virus (NDV)-based COVID-19 vaccine, in which a membrane-anchored spike protein is expressed on the surface of the NDV virion. This NDV-vector could be used either as a live vaccine or an inactivated vaccine $(2,3)$. Here we describe a next-generation

63 version of the NDV vector expressing a prefusion spike protein stabilized by HexaPro (HXP) mutations,

64 which are reported to contribute to high protein yield, favorable conformation and enhanced stability (4).

65 This construct is designated NDV-HXP-S. As an egg-based vaccine like the influenza virus vaccine, NDV-

66 HXP-S is suitable for large-scale production to cover a fair share of global demands. A survey conducted

67 by The World Health Organization (WHO) estimated the production capacity for pandemic influenza

68 vaccines (monovalent) could reach $\sim 4.15$ billion doses in 12 months by 31 established manufacturers 
worldwide, among which 28 manufacturers have egg-based facility producing $79 \%$ of total doses (5). This report realistically reflects the feasibility of manufacturing large quantify of NDV-based COVID-19 vaccine, since few modifications to the influenza virus vaccine manufacturing process are needed. Moreover, this estimation assumes the dose of NDV-based vaccine required will match that of monovalent pandemic influenza vaccines $(15 \mu \mathrm{g} / 0.5 \mathrm{~mL})$, without adjusting for the potential antigen-sparing effect of adjuvants (5). A safe and inexpensive adjuvant could likely expand the number of the doses per egg.

In the belief that NDV-HXP-S could be the solution for self-sufficient supplies of COVID-19 vaccine in many low- and middle-income countries (LMICs), in this study we thoroughly evaluated the immunogenicity and protective efficacy of live and inactivated NDV-HXP-S in preclinical mouse and Golden Syrian hamster models. We assessed the beta-propiolactone (BPL)-inactivated NDV-HXP-S whole-virion vaccine (GMP manufactured in Thailand, Vietnam and Brazil) via the intramuscular (IM) route using a two-dose regimen. In addition, we explored the possibility of using the NDV-HXP-S as a live vaccine either via the intranasal (IN) route or a combination of IN and IM routes. These preclinical studies showed that the NDV-HXP-S vaccine in its live or inactivated format was highly immunogenic, inducing potent binding and neutralizing antibodies (NAbs), which offered protections against SARS-CoV-2 replication or SARS-CoV-2 induced disease in vivo. The high levels of NAbs induced by NDV-HXP-S allowed the variants of concern/interest (B.1.1.7, B.1.351 or P.1) to be neutralized, despite the fact that a reduction of neutralization titer was observed against the B.1.351 variant. A similar level of reduction has been shown in other vaccine cohorts as well (6-9). Importantly, Good Manufacturing Practice (GMP)-grade NDV-HXP-S vaccine lots produced by manufacturers from Vietnam (Institute of Vaccines and Medical Biologicals, IVAC), Thailand (Government Pharmaceutical Organization, GPO) and Brazil (Instituto Butantan) showed excellent immunogenicity and protective efficacy in the hamster model, demonstrating the consistency of the manufacturing process in different locations and the possibility of mass production. Vaccine trials with the inactivated vaccines have been started in Thailand (NCT04764422, HXP-GPOVac) and Vietnam (NCT04830800, COVIVAC) and the live vaccine is currently in clinical development in Mexico (NCT04871737, Patria).

\section{Results}

\section{NDV expressing a membrane-bound pre-fusion stabilized spike protein}

To ensure high immunogenicity of the spike antigen expressed by the NDV vector, we improved the spike construct by introducing the pre-fusion stabilizing HexaPro (HXP) mutations that were identified and characterized in an earlier study (4). Specifically, we added the HXP mutations into our previously described S-F chimera (HXP-S), in which the polybasic cleavage site was removed and the transmembrane 
103 domain and cytoplasmic tail of the spike were replaced with those from the fusion (F) protein of NDV. The

104 nucleotide sequence of the construct was codon-optimized for mammalian host expression. The HXP-S

105 sequence was inserted between the P and M genes of the NDV genome and the virus was rescued (Fig.

106 1A). We concentrated the virus in the allantoic fluid through a sucrose cushion and performed a sodium

107 dodecyl-sulfate polyacrylamide gel electrophoresis (SDS-PAGE) to visualize NDV viral proteins as well

108 as the presence of the spike protein by Coomassie Blue staining. Compared to the WT NDV, the NDV-

109 HXP-S showed an extra band between $160 \mathrm{kD}$ and $260 \mathrm{kD}$ below the $\mathrm{L}$ protein of the NDV that corresponds

110 to the size of the uncleaved S0 (Fig. 1B).

Vaccination with inactivated NDV-HXP-S via the intramuscular route induces protective immune responses in mice and hamsters

To evaluate the immunogenicity and protective efficacy of the inactivated NDV-HXP-S, we first performed a dose-ranging study in mice that were "sensitized" by intranasal (IN) administration of a nonreplicating human adenovirus 5 expressing human angiotensin converting enzyme 2 (Ad5-hACE2). Mice are not naturally susceptible to SARS-CoV-2 infection, but gene delivery of hACE2 to the lungs of mice using a viral vector such as Ad5-hACE2, can sensitize mice to subsequent infection with SARS-CoV-2 (10).

BPL-inactivated NDV-HXP-S with or without an adjuvant CpG 1018 (11) was tested in the study. Specifically, we immunized BALB/c mice with low doses of NDV-HXP-S at a total protein content of 1 $\mu \mathrm{g}, 0.3 \mu \mathrm{g}, 0.1 \mu \mathrm{g}, 0.03 \mu \mathrm{g}$ and $0.01 \mu \mathrm{g}$ per mouse without the adjuvant. In the adjuvanted groups, mice 123 were vaccinated with either $0.1 \mu \mathrm{g}$ or $0.03 \mu \mathrm{g}$ of the vaccine in combination with $10 \mu \mathrm{g}$ or $30 \mu \mathrm{g}$ of $\mathrm{CpG}$ 1241018 per mouse. Mice that were immunized with $1 \mu \mathrm{g}$ of the inactivated WT NDV (vector-only) were used 125 as negative controls. The inactivated vaccine was administered via the intramuscular route following a 126 prime-boost regimen at a 3-week interval (Fig. 2A). Mice were bled to measure spike-specific serum IgG. 127 The prime immunization showed a dose-dependent antibody response, in which the adjuvanted groups 128 appeared to develop higher antibody responses than those which received the same amount of vaccine 129 without the adjuvant. The antibody titers were greatly enhanced after the boost in all the animals that were 130 vaccinated with NDV-HXP-S, resulting in no significant differences among the groups (with a marginal 131 dose-dependent trend). Of note, mice that were vaccinated with as low as $0.03 \mu \mathrm{g}$ and $0.01 \mu \mathrm{g}$ of vaccine 132 per animal also developed good IgG titers, suggesting the vaccine is highly immunogenic (Fig. 2B). Further 133 evaluation of IgG2a over IgG1 ratio in post-boost sera from 3 selected groups, group $1(1 \mu \mathrm{g})$ representing 134 animals that received the non-adjuvanted vaccine, group $7(0.1 \mu \mathrm{g}+30 \mu \mathrm{g} \mathrm{CpG} \mathrm{1018)} \mathrm{representing} \mathrm{animals}$ 135 that received the adjuvanted vaccine and group 10 (WT NDV) as the negative controls, suggested a $\mathrm{T}_{\mathrm{H}} 1$ 136 biased immune response (12). The $\mathrm{CpG} 1018$ appeared to reinforce a $\mathrm{T}_{\mathrm{H}} 1$-biased immune response, given 
137 that a more pronounced difference between the IgG2a and IgG1 levels was observed in the adjuvanted 138 group as compared to that in the non-adjuvanted group (Fig. S1A). To examine the neutralizing activity of 139 the immune sera from mice, pooled sera from group 1, 7 and 10 were tested. Neutralization titers against 140 the prototype (WT) USA-WA1/2020 virus as well as two SARS-CoV-2 variants, B.1.1.7 and B.1.351 were 141 measured (Fig. 2C). We observed potent neutralizing activity of mouse sera to the WT strain from group 1 $142 \quad\left(\mathrm{ID}_{50}=669\right)$ and group $7\left(\mathrm{ID}_{50}=627\right)$. As expected, mouse sera from both groups neutralized the B.1.1.7 143 variant equally well (group $1 \mathrm{ID}_{50}=652$; group $7 \mathrm{ID}_{50}=914$ ). An approximately five-fold reduction of 144 neutralization titers was observed toward the B.1.351 variant (group $1 \mathrm{ID}_{50}=132$; group $7 \mathrm{ID}_{50}=132$ ). Our 145 result is in agreement with decreased neutralization titers of mRNA vaccines that have been reported (6-9), 146 as the E484K mutation in the B.1.351 variant is mainly responsible for the resistance to NAbs $(13,14)$. To 147 assess protection conferred by vaccination, mice were treated with Ad5-hACE2 (10). Five days after the 148 Ad5-hACE2 treatment, mice were challenged with $10^{5}$ plaque forming unit (PFU) of USA-WA1/2020 149 SARS-CoV-2. Infectious viral titers in the lungs of challenged animals at day 2 and day 5 post-challenge 150 were measured as the readout of protection. We observed a significant drop of the viral titer in the lung 151 homogenates of all vaccinated animals compared to those from the negative control group, also in a dose152 dependent manner. At day 5 post-infection, infectious viruses were cleared in all NDV-HXP-S vaccinated 153 animals (Fig. 2D). These results support the conclusion that the inactivated NDV-HXP-S induced strongly 154 protective antibody responses directed to the spike protein of SARS-CoV-2, with CpG 1018 as a possible 155 dose-sparing adjuvant.

156 In the hamster model, we evaluated two-doses of inactivated NDV-HXP-S without adjuvants, with CpG 1018 or AddaVax as the adjuvant. Each hamster was vaccinated intramuscularly with a total of $5 \mu \mathrm{g}$ 158 of NDV-HXP-S. The negative control group was vaccinated with $5 \mu \mathrm{g}$ of inactivated WT NDV. The 159 healthy control group was kept unvaccinated. After two doses with a 3-week interval, hamsters were 160 challenged with $10^{4} \mathrm{PFU}$ of the USA-WA1/2020 strain, except that the healthy control group was mock161 challenged (Fig 3A). Change of body weight, viral titers in the lung homogenates and nasal washes at day 1622 and day 5 post-challenge were measured to evaluate protection. In addition, spike-specific IgG titers in 163 the post-prime and post-boost sera, as well as neutralizing activity in the post-boost sera were measured by 164 enzyme-linked immunosorbent assay (ELISA) and micro-neutralization assay, respectively. NDV-HXP-S 165 was found to be highly immunogenic in hamsters as well, with both CpG 1018 and AddaVax increasing 166 serum IgG (Fig 3B). Without any adjuvant, NDV-HXP-S vaccinated hamsters developed potent NAbs in 167 the post-boost sera $\left(\mathrm{ID}_{50}=2429\right)$ to the prototype (WT) SARS-CoV-2. AddaVax enhanced NAb titers $\left(\right.$ ID $_{50}$ 168 = 3913), but the $\mathrm{CpG} 1018$ did not $\left(\mathrm{ID}_{50}=970\right)$. Interestingly, the antibodies in the $\mathrm{CpG} 1018$ group 169 appeared to be more cross-neutralizing to B.1.351 with only a 2-fold decrease $\left(\mathrm{ID}_{50}=480\right)$. A $\sim 6$-fold 170 reduction of neutralizing titer to the B.1.351 $\left(\mathrm{ID}_{50}=425\right)$ was observed in the unadjuvanted group, while a 
$171 \sim 4$-fold reduction of neutralizing activity to the B.1.351 $\left(\mathrm{ID}_{50}=1144\right)$ was observed in the AddaVax group

172 (Fig 3C). After challenge, animals in both adjuvanted groups showed quicker recovery of the body weight 173 than animals in the unadjuvanted group (Fig 3D). Viruses were cleared in the lungs of all NDV-HXP-S

174 vaccinated hamsters at day 2 except for one out of four animals in the unadjuvanted and CpG 1018 group 175 which showed low titers that were very close to the limit of detection. Viral load became undetectable at 176 day 5 post-challenge in all NDV-HXP-S vaccinated hamsters. In addition, all NDV-HXP-S vaccinated 177 groups showed a reduction of viral titers in the nasal washes compared to control animals (Fig 3E). In 178 conclusion, the inactivated NDV-HXP-S induced high levels of binding and neutralizing antibody 179 responses in hamsters, significantly reduced viral titers in the lungs and lowered virus shedding from the nasal cavity. Both CpG 1018 and AddaVax exhibited beneficial adjuvant effects.

\section{Formulations of inactivated NDV-HXP-S GMP-produced by GPO, IVAC and Instituto Butantan} are effective in a preclinical hamster study

In the belief that the existing influenza virus vaccine manufacturers should be equipped to produce manufacturers (IVAC, GPO, Instituto Butantan) from Vietnam, Thailand and Brazil, pilot GMP lots of whole BPL- inactivated NDV-HXP-S vaccines were produced. We obtained these vaccine preparations and subsequently evaluated them in hamsters. Vaccines containing $1 \mu \mathrm{g}$ of $\mathrm{S}$ antigen were administered intramuscularly to each hamster with or without $100 \mu \mathrm{g} \mathrm{CpG} 1018$ as the adjuvant following a prime-boost regimen with a 3-week interval. A PBS-vaccinated negative control and a healthy control group were included. Two weeks after the boost, animals were challenged with $10^{4} \mathrm{PFU}$ of the USA-WA1/2020 strain (Fig. 4A). Animals having received vaccines from different producers developed comparable binding IgG titers, while $\mathrm{CpG} 1018$ showed marginal adjuvant effects (Fig. 4B). The neutralizing activity of serum antibodies after the boost was measured in a pseudo-particle neutralization assay at Nexelis (part of CEPI's global network of laboratories to centralize assessment COVID-19 vaccine candidates) (15). Neutralization titers of hamster sera were substantially higher than those of human convalescent sera used as controls in

198 vaccines without the adjuvant developed a similar trend of body weight change. Animals that were 199 immunized with GPO and Butantan vaccines in the presence of the CpG 1018 showed improved protection 200 manifested by less weight loss. The adjuvant did not seem to alleviate body weight loss of animals that 201 received the IVAC vaccine (Fig. 4D). This observation is consistent with the post-boost antibody level 202 (D33) (Fig. 4B). Nevertheless, all the animals that were vaccinated with NDV-HXP-S developed immunity 203 to inhibit SARS-CoV-2 replication in the lungs showing no detectable infectious viral titers (Fig. 4E). In 204 the nasal washes of vaccinated animals, virus shedding was significantly reduced in contrast to that in the 
negative control animals (Fig. 4F). Virus was still able to replicate in the nasal turbinates of all infected animals, but all NDV-HXP-S vaccinated animals showed a reduced viral load (Fig. 4G). To evaluate SARS-CoV-2 induced lung disease, the left lung lobes were collected at day 5 post-challenge and processed for histopathology analysis. As expected, much less pathological change reflecting injury or inflammation was observed in the lungs of NDV-HXP-S vaccinated animals as compared to those in the lungs of negative control animals (Fig. S2). In conclusion, inactivated NDV-HXP-S vaccine prepared by three eggbased influenza virus vaccine manufacturers showed equally good efficacy at inducing binding/neutralizing antibodies, inhibiting virus replication and shedding as well as minimizing SARS-CoV-2 induced lung pathology in hamsters. An FDA approved adjuvant, CpG 1018 mildly increased protection from body weight loss of hamsters after challenge.

\section{Live NDV-HXP-S protects hamsters and mice from SARS-CoV-2 challenge}

While there is no attenuated SARS-CoV-2 available as a live vaccine, live viral vector COVID-19 vaccines were rapidly developed in several platforms. In addition to adenovirus vectors, paramyxovirus vectors have been used such as measles virus (16) and Newcastle disease virus (2, 3, 17). Live vaccines typically would have an advantage over inactivated vaccine at inducing strong local innate immune responses, T-cell responses and sterilizing mucosal antibody responses - especially when administered mucosally. To reiterate that the design of the NDV-HXP-S renders versatility of the construct to be used as both inactivated and live vaccine, we evaluated NDV-HXP-S as a live vector vaccine in two preclinical animal models testing two different vaccination regimens. First, we examined two immunizations of live NDV-HXP-S via the intranasal route in the hamster model. NDV-HXP-S at a dose of $10^{6}$ fifty percentage of egg embryo infectious dose $\left(\mathrm{EID}_{50}\right)$ administered to hamsters intranasally twice at day 0 and day 22 . A vector only control group was immunized with the same dose of WT NDV. A negative control group was mock-vaccinated with PBS. A healthy control group was kept unvaccinated (Fig. 5A). Serum IgG titer showed that one immunization was sufficient to induce potent binding antibody responses, while the booster vaccination did not further increase titers. We speculated that the booster vaccination might generate more mucosal immunity such as spike-specific IgA, which was not measured in this study (Fig. 5B). The neutralizing activity of post-boost sera was measured against the (WT) USA-WA1/2020, and the B.1.351 and B.1.1.7 variants. A similar trend was observed as in previous studies, where immune sera neutralized the (WT) USA-WA1/2020 and the B.1.1.7 variant equally well and crossed-neutralized the B.1.351 variant with a reduced potency (Fig. 5C). Upon challenge with $10^{5} \mathrm{PFU}$ of the USA-WA1/2020 strain, we observed

236 that the NDV-HXP-S vaccinated animals show no weight changes like the healthy control group, whereas

237 the negative control group lost a substantial amount of weight by day 5. Animals vaccinated with the WT

238 NDV exhibited weight loss that was less pronounced than that of the negative control group. This could 
possibly be due to the innate antiviral response induced by the live WT NDV (Fig. 5D). At day 2 post infection, viruses were cleared in the lungs of animals that received the NDV-HXP-S, whereas both WT NDV and PBS groups showed similar high viral titers in the lung homogenates. In the nasal washes of WT

242 NDV and PBS control animals, viral titers are comparably high, while only one animal out of three in the

243 NDV-HXP-S group showed measurable viral titer (Fig. 5E). In summary, this study confirmed the

244 effectiveness of the NDV-HXP-S as a live viral vector vaccine when administered intranasally. The vaccine

245 not only prevented SARS-CoV-2 replication in the lungs but also significantly reduced virus shedding from

246 the nasal cavity, which would greatly diminish the risk of virus transmission as well.

In addition to the hamster study testing only the intranasal route of the live vaccine, we performed a mouse study evaluating a different immunization regimen of the live NDV-HXP-S, combining the intranasal and intramuscular routes aiming to bring both mucosal and systemic immunity into action. Of note, the live nature of the NDV-HXP-S would have an adjuvant effect compared to an inactivated NDVHXP-S. Here we examined this vaccination strategy with three different doses, in which the animals received the same titer of live NDV-HXP-S for the intranasal prime and the intramuscular boost. Three groups of animals were immunized with $10^{4} \mathrm{EID}_{50}, 10^{5} \mathrm{EID}_{50}$ and $10^{6} \mathrm{EID}_{50}$ of NDV-HXP-S, respectively. A vector-only control group was immunized with $10^{6}$ EID $_{50}$ of the WT NDV. The negative control group was mock-vaccinated with PBS. The two immunizations were 3 weeks apart. Mice were again sensitized with Ad5-hACE2 as described earlier and challenged with $10^{5}$ PFU of the USA-WA1/2020 strain (Fig. 6A). By ELISA we observed a dose-dependent antibody titer, in which the high-dose group developed the strongest antibody responses after each immunization (Fig. 6B). IgG subclasses ELISAs showed a favorable induction of IgG2a over IgG1 in all NDV-HXP-S vaccine groups (Fig. S1B). As expected, mice vaccinated with the high-dose developed the highest level of neutralizing/cross-neutralizing antibodies to the WT and variant SARS-CoV-2 (Fig. 6C). In terms of protection, a reverse correlation of the vaccine dose and viral load in the lung homogenates was observed (Fig. 6D); the high-dose regimen conferred the best protection among the three groups. A duplicate experiment including the high dose-group $\left(10^{6} \mathrm{EID}_{50}\right.$ of NDV-HXP-S) and vector-only control group ( $10^{6} \mathrm{EID}_{50}$ of WT NDV) were set up to measure spikespecific IgA in the nasal washes, which were collected 21 days after intranasal prime. A spike-specific IgA ELISA showed that animals which received the NDV-HXP-S developed IgA on their respiratory mucosal surfaces (Fig. S3).

\section{NDV-HXP-S is effective against SARS-CoV-2 variants of concern in the mouse model}

With the emergence of variants of concern that are partially resistant to NAbs raised against the WT SARS-CoV-2 attributed to the amino acid substations or deletions in the N-terminal domain (NTD) and the receptor-binding domain (RBD), we performed vaccination in mice with inactivated NDV-HXP-S 
and challenged them with USA-WA1/2020, hCoV-19/USA/MD-HP01542/2021 JHU (B.1.351) and hCoV19/Japan/TY7-503/2021 (P.1) after Ad5-hACE2 treatment. This was to ensure efficient replication of all three viruses, although both B.1.351 and P.1 variants have been reported to extend their host tropism to murine ACE2 (18). The vaccination group received intramuscular injection of $1 \mu \mathrm{g}$ of inactivated NDVHXP-S, while the negative control group received $1 \mu \mathrm{g}$ of the inactivated WT NDV. Two doses were administered, 3 weeks apart. One third of the mice from each group was challenged with USA-WA1/2020, hCoV-19/USA/MD-HP01542/2021 JHU (B.1.351) and hCoV-19/Japan/TY7-503/2021 (P.1), respectively. Lungs of animals from each group were harvested at day 2. Viral loads were measured as described earlier. The NDV-HXP-S reproducibly inhibited WT virus replication to a great magnitude, while it also reduced B.1.351 replication by a factor of $\sim 1000$ and P.1 replication by a factor of $\sim 280$ at day 2 post-challenge

(Fig 7). This study demonstrated that with expected reduction to neutralize the variants of concern, NDVHXP-S is still effective at robustly inhibiting virus replication in vivo, which would be essential to mitigate disease.

\section{Discussion}

The COVID-19 pandemic has promoted the unprecedented development of various vaccine platforms from the conventional inactivated whole-virion vaccines, to novel mRNA vaccines, recombinant protein subunit vaccines and viral vector vaccines (19). The high efficacy of some novel vaccines is not necessarily followed by universal accessibility, due to logistical barriers (e.g. cost, manufacturing infrastructure, supply of raw materials, production capacity, transportation/storage and distribution). Newcastle disease virus was identified as an avian pathogen in the 1920's, and was later developed as oncolytic agent and veterinary vaccine using non-virulent strains (20-25). Similar to many other paramyxoviruses, NDV tolerates large insertions and has been evaluated as vaccine vector for a number of animal pathogens in preclinical studies $(22,25-33)$. To increase the surface expression of the inserted gene, the transmembrane domain and cytoplasmic tail of the target membrane protein can be replaced by that of $\mathrm{HN}$ or F protein of NDV (32). Using this strategy, we have previously constructed a viral vector (NDV expressing the S-F chimera) and shown that it can be used as an inactivated vaccine against SARS-CoV-2 (2). This construct was later optimized to further stabilize the S-F by introducing HexaPro mutations identified via structural biology (34). This second generation NDV-HXP-S was quickly developed and is currently being evaluated in Phase I clinical trials in Mexico (NCT04871737, live vaccine), Vietnam (NCT04830800, inactivated vaccine) and Thailand (NCT04764422, inactivated vaccine).

While the clinical trials are ongoing, we demonstrate here the versatility of the NDV-HXP-S in 
vaccine. The live nature of the vaccine allowed for mucosal administration as well as intramuscular administration. There is also an important manufacturing consideration differentiating the live NDV vaccine from the inactivated vaccine technology. Live vaccine for humans must be propagated in specific pathogen free (SPF) embryonated eggs, while inactivated vaccine for humans can be safely manufactured in non-SPF eggs, as the inactivating process eliminates the risk of avian adventitious agents arising from

312 the use of widely available breeder chicken eggs that are one tenth the cost and not limited in supply. A

313 dose-ranging study in mice indicated the inactivated NDV-HXP-S was highly immunogenic even at a dose

314 as low as $0.01 \mu \mathrm{g}$ of the total protein (the amount of the spike protein would be much less). The adjuvant

315 CpG 1018 leads to an antigen-sparing effect which is slightly better in mice than that in hamsters. It was

316 postulated that the NDV could be self-adjuvanting due to viral components (RNA and proteins) being

317 present, resulting in less beneficial effect of the adjuvant. However, we did observe that CpG 1018 drove a

318 more prominent IgG2a production over IgG1 than the unadjuvanted vaccine in mice, suggesting it could

319 promote a favorable $\mathrm{T}_{\mathrm{H}} 1$ response, which will be measured in the Phase I trials that include CpG 1018

320 adjuvanted groups. The final verdict for the usefulness of CpG 1018 in combination with NDV-HXP-S will

321 come from the human data. Moreover, it appeared that an oil-in-water nano-emulsion adjuvant (AddaVax)

322 also enhanced antibody titers. It will be of great interest to evaluate other adjuvants in our future preclinical 323 studies.

324 It was observed that mice and hamsters vaccinated with NDV-HXP-S developed strong antibody responses that not only neutralized the prototype SARS-CoV-2 but also cross-neutralized variants of interest/concern. The reduction of neutralizing activity against B.1.351 and B.1.1.7 is consistent with what was observed for other vaccines using the prototype spike as the immunogen (6-9). Interestingly, crossneutralization was improved by using CpG 1018 as adjuvant. Challenge studies in Ad5-hACE2 sensitized mice using B.1.351 and P.1 variants demonstrated good protection by the prototype antigen expressed by the NDV. Last but not least, this NDV platform could be quickly adapted to express the spike protein of SARS-CoV-2 variants. So far, we have successfully generated NDV-HXP-S (B.1.351), NDV-HXP-S (B.1.1.7) and NDV-HXP-S (P.1) and are evaluating them in animal models. Heterologous vaccination regimens or multi-valent formulations might be beneficial to the induction of cross-protective antibodies.

336 Materials and methods

337 Cells

BSRT7 cells were a kind gift from Dr. Benhur Lee at Icahn School of Medicine at Mount Sinai (ISMMS) $(35,36)$ and Vero E6 cells were purchased from ATCC (CRL-1586). Chicken embryo fibroblasts (CEF) were isolated as described in a previous study (37). All cell lines were maintained in Dulbecco's 
Modified Eagle's Medium (DMEM; Gibco) containing 10\% (vol/vol) fetal bovine serum (FBS), 100 unit/mL of penicillin, $100 \mu \mathrm{g} / \mathrm{mL}$ of streptomycin (P/S; Gibco) and $10 \mathrm{mM} \mathrm{4-(2-hydroxyethyl)-1-}$ piperazineethanesulfonic acid (HEPES) at $37^{\circ} \mathrm{C}$ with $5 \% \mathrm{CO} 2$.

\section{Plasmids}

HexaPro (HXP) mutations including F817P, A892P, A899P, A942P, K986P and V987P that have been identified to stabilize the prefusion conformation of spike (4) were introduced into the S-F chimera by PCR (HXP-S) (2). The sequence of the HXP-S was inserted into pNDV_LS/L289A rescue plasmid (between $\mathrm{P}$ and $\mathrm{M}$ genes) by in-Fusion cloning (Clontech). The recombination product was transformed into MAX Efficiency ${ }^{\mathrm{TM}} \mathrm{Stb} 12^{\mathrm{TM}}$ Competent Cells (Thermo Fisher Scientific) to generate the pNDV-HXP$\mathrm{S}$ rescue plasmid. The plasmid was purified using PureLink ${ }^{\mathrm{TM}}$ HiPure Plasmid Maxiprep Kit (Thermo Fisher Scientific).

\section{Rescue of the NDV-HXP-S}

As described in our previous studies (2), BSRT7 cells stably expressing the T7 polymerase were seeded onto 6-well plates at $3 \times 10^{5}$ cell per well in duplicate. The next day, cells were transfected with 2 $\mu \mathrm{g}$ of pNDV-HXP-S, $1 \mu \mathrm{g}$ of pTM1-NP, $0.5 \mu \mathrm{g}$ of pTM1-P, $0.5 \mu \mathrm{g}$ of pTM1-L and $1 \mu \mathrm{g}$ of pCI-T7opt were re-suspended in $250 \mu \mathrm{L}$ of Opti-MEM (Gibco). The plasmid cocktail was then gently mixed with $15 \mu \mathrm{L}$ of TransIT LT1 transfection reagent (Mirus). The growth media were replaced with opti-MEM during transfection. To increase rescue efficiency, BSRT7-CEF co-culture was established the next day as described previously (38). Specifically, transfected BSRT7 cells and CEF wells were washed with warm PBS and trypsinized. Trypsinized cells were neutralized with excessive amount of growth media. Mix BSRT7 cells with CEF cells ( 1: 2.5) in a 10-cm dish. The co-culture was incubated at $37^{\circ} \mathrm{C}$ overnight. The next day, the media were removed and cells were gently washed with warm PBS, opti-MEM supplemented with $1 \% \mathrm{P} / \mathrm{S}$ and $0.1 \mu \mathrm{g} / \mathrm{mL}$ of tosyl phenylalanyl chloromethyl ketone (TPCK)- treated trypsin was added. The co-cultures were incubated for 2 or 3 days before inoculation into 8 or 9 day-old embryonated chicken eggs. To inoculate eggs, cells and supernatants were harvested and homogenized by several syringe strokes. One or two hundred microliters of the mixture was injected into each egg. Eggs were incubated at $37^{\circ} \mathrm{C}$ for 3 days and cooled at $4^{\circ} \mathrm{C}$ overnight. Allantoic fluid was harvested from cooled eggs and the rescue of the viruses was determined by hemagglutination (HA) assays. 
The viruses in the allantoic fluid were first inactivated using $0.05 \%$ beta-propiolactone (BPL) as described previously (2). To concentrate the viruses, allantoic fluids were clarified by centrifugation at $3764,000 \mathrm{rpm}$ at $4^{\circ} \mathrm{C}$ for $30 \mathrm{~min}$ using a Sorvall Legend RT Plus Refrigerated Benchtop Centrifuge (Thermo 377 Fisher Scientific). Clarified allantoic fluids were laid on top of a 20\% sucrose cushion in NTE buffer (100 $378 \mathrm{mM} \mathrm{NaCl}, 10 \mathrm{mM}$ Tris-HCl, 1 mM EDTA, $\mathrm{pH}$ 7.4). Ultracentrifugation in a Beckman L7-65 ultracentrifuge at 25,000 rpm for two hours at $4^{\circ} \mathrm{C}$ using a Beckman SW28 rotor (Beckman Coulter) was performed to pellet the viruses through the sucrose cushion while soluble egg proteins were removed. The virus pellets were re-suspended in PBS ( $\mathrm{pH}$ 7.4). The total protein content was determined using the bicinchoninic acid (BCA) assay (Thermo Fisher Scientific).

\section{SDS-PAGE}

The concentrated NDV-HXP-S or WT NDV was mixed with Novex ${ }^{\mathrm{TM}}$ Tris-Glycine SDS Sample Buffer (2X) (Thermo Fisher Scientific), NuPAGE ${ }^{\mathrm{TM}}$ Sample Reducing Agent $(10 \mathrm{x})$ (Thermo Fisher Scientific) and PBS at appropriate amounts to reach a total protein content of $20 \mu \mathrm{g}$ in $50 \mu 1$ volume. The mixture was heated at $90{ }^{\circ} \mathrm{C}$ for $5 \mathrm{~min}$. The samples were mixed by pipetting and loaded at $30 \mu \mathrm{g}$ to a 420\% 10-well Mini-PROTEAN TGX ${ }^{\mathrm{TM}}$ precast gel (Bio-Rad). Ten microliters of the Novex ${ }^{\mathrm{TM}}$ Sharp Prestained Protein standard (Thermo Fisher Scientific) was used as the ladder. The electrophoresis was run in Tris/Glycine SDS/Buffer (Bio-Rad). The gel was then washed with distilled water at room temperature several times until the dye front in the gel was no longer visible. The gel was stained with $20 \mathrm{~mL}$ of SimplyBlue $^{\mathrm{TM}}$ SafeStain (Thermo Fisher Scientific) for a minimal of 1 hour to overnight. The SimplyBlue ${ }^{\mathrm{TM}}$ SafeStain was decanted and the gel was washed with distilled water several times until the background was clear. Gels were imaged using the Bio-Rad Universal Hood Ii Molecular imager (Bio-Rad) and processed by Image Lab Software (Bio-Rad).

\section{Virus titration by EID $_{50}$ assays}

Fifty percent of egg embryo infectious dose $\left(\mathrm{EID}_{50}\right)$ assay was performed in 9 to 11 -day old chicken

402 dilutions of the virus. One hundred microliters of each dilution was injected into each egg for a total of 5-

40310 egg per dilution. The eggs were incubated at $37^{\circ} \mathrm{C}$ for 3 days and then cooled at $4{ }^{\circ} \mathrm{C}$ overnight. Allantoic 404 fluids were collected and analyzed by HA assay. The EID ${ }_{50}$ titer of the NDV, determined by the number of 405 HA-positive and HA-negative eggs in each dilution was calculated using the Reed and Muench method.

\section{Animal experiments}


All the animal experiments were performed in accordance with protocols approved by the Icahn School of Medicine at Mount Sinai Institutional Animal Care and Use Committee (IACUC). All experiments with live SARS-CoV-2 were performed in the Centers for Disease Control and Prevention (CDC)/US Department of Agriculture (USDA)-approved biosafety level 3 (BSL-3) biocontainment facility of the Global Health and Emerging Pathogens Institute at the Icahn School of Medicine at Mount Sinai, in accordance with institutional biosafety requirements.

\section{Mouse immunization and challenge studies}

Female BALB/c mice were used in all studies. For intramuscular vaccination using the inactivated NDV-HXP-S, vaccine or negative control WT NDV was prepared in $100 \mu 1$ total volume with or without CpG 1018 as the adjuvant. Two immunizations were performed for all the mice with a 21-day interval. To administer live NDV-HXP-S, mice were anesthetized with ketamine/xylazine cocktail and vaccinated with NDV-HXP-S, WT NDV or PBS in $30 \mu 1$ total volume via the intranasal route (IN) and boosted with the same preparation via the IM route with a 21-day interval. For SARS-CoV-2 infection, mice were intranasally infected with $2.5 \times 10^{8}$ PFU of Ad5-hACE2 5 days prior to being challenged with $10^{5} \mathrm{PFU}$ of the USA-WA1/2020 strain, $3.4 \times 10^{4} \mathrm{PFU}$ of the hCoV-19/USA/MD-HP01542/2021 JHU strain (B.1.351, kindly provided by Dr. Andrew Pekosz from Johns Hopkins Bloomberg School of Public Health) or $6.3 \mathrm{x}$ $10^{4} \mathrm{PFU}$ of the hCoV-19/Japan/TY7-503/2021 strain (P.1). Viral titers in the lung homogenates of mice 2 days or 5 days post-infection were used as the readout for protection. Briefly, the lung lobes were harvested from a subset of animals per group and homogenized in $1 \mathrm{~mL}$ of sterile PBS. Viral titers in the lung homogenates were measured by plaque assay on Vero E6 cells. To collect nasal washes, mice were euthanized and nasal washes were collected in $1 \mathrm{~mL}$ PBS containing $0.1 \%$ BSA, 10 units $/ \mathrm{mL}$ penicillin and $10 \mu \mathrm{g} / \mathrm{mL}$ streptomycin. The nasal washes were spun at $3,000 \mathrm{rpm}$ for $20 \mathrm{~min}$ at $4{ }^{\circ} \mathrm{C}$ and stored at $-80^{\circ} \mathrm{C}$. Blood was collected by submandibular vein bleeding. Sera were isolated by low-speed centrifugation and stored at $-80^{\circ} \mathrm{C}$ until use.

\section{Hamster immunization and challenge studies}

Female Golden Syrian hamsters were used in all the studies. For intramuscular vaccination study, NDV-HXP-S vaccine or negative control WT NDV/PBS was prepared in $100 \mu \mathrm{L}$ of total volume either without adjuvants, or with $100 \mu \mathrm{g}$ of $\mathrm{CpG} 1018$ or $50 \mu \mathrm{L}$ of AddaVax as the adjuvant. For the intranasal vaccination study, hamsters were anesthetized with ketamine/xylazine cocktail before the intranasal administration of live NDV-HXP-S, WT NDV or PBS in a $50 \mu \mathrm{L}$ volume. An unvaccinated healthy control group was included in each study. The animals were vaccinated following a prime-boost regimen in a $\sim 3$ week interval. Two to three-weeks after the boost, animals were challenged with $10^{4}$ or $10^{5} \mathrm{PFU}$ of the 
USA-WA1/2020 strain, except that the healthy control group was mock-challenged with the same amount of PBS. Animals were bled via lateral saphenous vein and sera were isolated by low-speed centrifugation.

444 Weight changes of the animals were monitored for 5 days. A subset of animals from each group was

445 euthanized at day 2 and day 5 post-challenge to harvest lungs lobes (upper right lung lobe, lower right lung 446 lobe), nasal turbinates or nasal washes. Each right lung lobe was homogenized in $1 \mathrm{~mL}$ of PBS. The nasal 447 turbinates were homogenized in $0.5 \mathrm{~mL}$ of PBS. Nasal washes were collected in $0.4 \mathrm{~mL}$ PBS. Viral titers 448 in the nasal washes, nasal turbinate and lung homogenates were measured by plaque assay on Vero E6 cells.

449 The left lung lobes were collected at day 5 and fixed/perfused with neutral buffered formalin for 450 histopathology. Of note, the challenge study using GMP vaccine from the three collaborating manufacturers 451 was conducted using similar methods for assessments of outcomes, but with vaccine formulations from 452 each manufacturer containing $1 \mu \mathrm{g}$ of S protein per dose, with or without $\mathrm{CpG} 1018$.

\section{ELISAs}

ELISAs were performed as described previously $(2,3)$ to measure spike-specific IgG in the serum of mice and hamsters vaccinated with NDV-HXP-S. To measure spike-specific IgG1 or IgG2a subclass in the mouse sera, an HRP-conjugated goat anti-mouse IgG1 (ab97240, Abcam) or an HRP-conjugated goat anti-mouse IgG2a (ab97245, Abcam) were used at 1:3000 dilution. A starting dilution of 1: 30 was used for serum samples. To measure spike-specific IgA in the nasal wash, the clarified undiluted nasal washes were used and serially diluted by 2 -fold. An HRP-conjugated goat anti-mouse IgA secondary antibody (PA1-74397 Invitrogen) was used at 1: 2000 dilution.

\section{Microneutralization assays using the authentic SARS-CoV-2 viruses}

The microneutralization assays using the authentic SARS-CoV-2 viruses were described previously (39). To compare the neutralization titers of immune sera from animals vaccinated with NDVHXP-S, the neutralization assays against the wild type SARS-CoV-2 (isolate USA-WA1/2020), hCoV19/South Africa/KRISP-K005325/2020 (B.1.351, BEI Resources NR-54009) and hCoV19/England/204820464/2020 (B.1.1.7, BEI Resources NR-54000) were performed at the same time to avoid assay-to-assay variations.

\section{Pseudo-particle neutralization assays}

The pseudo-particle neutralization assay (PNA) was performed by Nexelis using a replication incompetent vesicular stomatitis virus (VSV) displaying the spike protein of Wuhan-Hu-1 strain (15). 
The plaque assay was performed in BSL3 facility. Vero E6 cells were seeded onto 12-well plates

477 in growth media at 1:5 and were cultured for two days. Tissue homogenates or nasal washes were 10-fold 478 serially diluted in infection medium (DMEM containing 2\% FBS, P/S and $10 \mathrm{mM}$ HEPES). Two hundred 479 microliters of each dilution were inoculated onto each well starting with 1:10 dilution of the sample. The 480 plates were incubated at $37^{\circ} \mathrm{C}$ for 1 hour with occasional rocking every 10 minutes. The inoculum in each 481 well was then removed and $1 \mathrm{~mL}$ of agar overlay containing $0.7 \%$ of agar in $2 \times$ MEM was placed onto 482 each well. Once the agar was solidified, the plates were incubated at $37{ }^{\circ} \mathrm{C}$ with $5 \% \mathrm{CO}_{2}$. Two days later, 483 the plates were fixed with 5\% formaldehyde in PBS overnight before being taken out of BSL3 for 484 subsequent staining in BSL2 cabinet. The plaques were immuno-stained with an anti-SARS-CoV-2 NP 485 primary mouse monoclonal antibody 1C7C7 kindly provided by Dr. Thomas Moran at ISMMS. An HRP486 conjugated goat anti-mouse secondary antibody was used at 1:2000 and the plaques were visualized using 487 TrueBlue ${ }^{\mathrm{TM}}$ Peroxidase Substrate (SeraCare Life Sciences Inc.).

$491 \quad$ Formalin-fixed, paraffin-embedded (FFPE) left lung tissues obtained from hamsters were cut into $4925 \mu \mathrm{m}$ sections and stained with hematoxylin and eosin (H\&E) by the Biorepository and Pathology Core. 493 All sections were evaluated by a veterinary pathologist who was blinded to the vaccination groups in the 494 Comparative Pathology Laboratory (CPL) at ISMMS. The scoring system used was described in the table 495 below.

\begin{tabular}{|c|c|c|c|}
\hline Score & Area affected & $\begin{array}{l}\text { Epithelial } \\
\text { degeneration/necrosis }\end{array}$ & Inflammation \\
\hline 0 & none & none & None \\
\hline 1 & $5-10 \%$ & $\begin{array}{l}\text { Minimal; scattered cell } \\
\text { necrosis/vacuolation affecting } 5 \\
\text { to } 10 \% \text { of tissue section }\end{array}$ & $\begin{array}{l}\text { Minimal; scattered } \\
\text { inflammatory cells } \\
\text { affecting } 5-10 \% \text { of } \\
\text { tissue section }\end{array}$ \\
\hline 2 & $10-25 \%$ & $\begin{array}{l}\text { Mild; scattered cell } \\
\text { necrosis/vacuolation }\end{array}$ & $\begin{array}{l}\text { multifocal, few } \\
\text { inflammatory cells }\end{array}$ \\
\hline 3 & $25-50 \%$ & $\begin{array}{l}\text { Moderate; multifocal vacuolation } \\
\text { or sloughed/necrotic cells }\end{array}$ & $\begin{array}{l}\text { Thin layer of cells }(<5 \\
\text { cell layer thick) }\end{array}$ \\
\hline
\end{tabular}




\begin{tabular}{|l|l|l|l|}
\hline 4 & $50-75 \%$ & $\begin{array}{l}\text { Marked; multifocal/segmental } \\
\text { necrosis, epithelial } \\
\text { loss/effacement }\end{array}$ & $\begin{array}{l}\text { Thick layer of cells }(>5 \\
\text { cell layer thick })\end{array}$ \\
\hline 5 & $>75 \%$ & $\begin{array}{l}\text { Severe; coalescing areas of } \\
\text { necrosis, parenchymal } \\
\text { effacement }\end{array}$ & $\begin{array}{l}\text { Confluent areas of } \\
\text { inflammation }\end{array}$ \\
\hline
\end{tabular}

Statistical analysis

The statistical analysis was performed using GraphPad Prism 7.0. For multiple comparison, the statistical difference was determined using ordinary one-way ANOVA or two-way ANOVA with Dunnett's correction. To compare two groups, a one-tailed $t$ test was used.

\section{Data availability}

The data that support the findings of this study are available from the corresponding author upon reasonable request

\section{References}

514 1. Anonymous. 2021. Tracking Coranavirus Vacination Around the World. The New York Times.

515 2. Sun W, McCroskery S, Liu WC, Leist SR, Liu Y, Albrecht RA, Slamanig S, Oliva J, Amanat F,

3. Sun W, Leist SR, McCroskery S, Liu Y, Slamanig S, Oliva J, Amanat F, Schafer A, Dinnon KH,

4. Hsieh CL, Goldsmith JA, Schaub JM, DiVenere AM, Kuo HC, Javanmardi K, Le KC, Wrapp D, Schafer A, Dinnon KH, 3rd, Innis BL, Garcia-Sastre A, Krammer F, Baric RS, Palese P. 2020. A 3rd, Garcia-Sastre A, Krammer F, Baric RS, Palese P. 2020. Newcastle disease virus (NDV) expressing the spike protein of SARS-CoV-2 as a live virus vaccine candidate. EBioMedicine 62:103132. 
6. Shen X, Tang H, Pajon R, Smith G, Glenn GM, Shi W, Korber B, Montefiori DC. 2021. Neutralization of SARS-CoV-2 Variants B.1.429 and B.1.351. N Engl J Med doi:10.1056/NEJMc2103740.

533 7. Liu Y, Liu J, Xia H, Zhang X, Fontes-Garfias CR, Swanson KA, Cai H, Sarkar R, Chen W,

8. Zhou D, Dejnirattisai W, Supasa P, Liu C, Mentzer AJ, Ginn HM, Zhao Y, Duyvesteyn HME, Cutler M, Cooper D, Weaver SC, Muik A, Sahin U, Jansen KU, Xie X, Dormitzer PR, Shi PY.

9. Hoffmann M, Arora P, Gross R, Seidel A, Hornich BF, Hahn AS, Kruger N, Graichen L, escape from neutralizing antibodies. Cell 184:2384-2393 e12.

10. Rathnasinghe R, Strohmeier S, Amanat F, Gillespie VL, Krammer F, Garcia-Sastre A, Coughlan L, Schotsaert M, Uccellini MB. 2020. Comparison of transgenic and adenovirus hACE2 mouse models for SARS-CoV-2 infection. Emerg Microbes Infect 9:2433-2445.

11. Campbell JD. 2017. Development of the CpG Adjuvant 1018: A Case Study. Methods Mol Biol 1494:15-27.

12. Stevens TL, Bossie A, Sanders VM, Fernandez-Botran R, Coffman RL, Mosmann TR, Vitetta ES. 1988. Regulation of antibody isotype secretion by subsets of antigen-specific helper T cells. Nature 334:255-8.

14. Xie X, Liu Y, Liu J, Zhang X, Zou J, Fontes-Garfias CR, Xia H, Swanson KA, Cutler M, Cooper

13. Jangra S, Ye C, Rathnasinghe R, Stadlbauer D, Personalized Virology Initiative study g, Krammer F, Simon V, Martinez-Sobrido L, Garcia-Sastre A, Schotsaert M. 2021. SARS-CoV-2 spike E484K mutation reduces antibody neutralisation. Lancet Microbe doi:10.1016/S26665247(21)00068-9. D, Menachery VD, Weaver SC, Dormitzer PR, Shi PY. 2021. Neutralization of SARS-CoV-2 spike 69/70 deletion, E484K and N501Y variants by BNT162b2 vaccine-elicited sera. Nat Med 27:620-621. 
563 15. Kumar A, Bernasconi V, Manak M, de Almeida Aranha AP, Kristiansen PA. 2021. The CEPI centralised laboratory network: supporting COVID-19 vaccine development. Lancet 397:21482149.

16. Lu M, Dravid P, Zhang Y, Trivedi S, Li A, Harder O, Kc M, Chaiwatpongsakorn S, Zani A,

17. Rohaim MA, Munir M. 2020. A Scalable Topical Vectored Vaccine Candidate against SARS-

18. Xavier Montagutelli MP, Laurine Levillayer, Eduard Baquero Salazar, Grégory Jouvion, Laurine

19. Krammer F. 2020. SARS-CoV-2 vaccines in development. Nature doi:10.1038/s41586-020-27983.

20. Vijayakumar G, Zamarin D. 2020. Design and Production of Newcastle Disease Virus for Intratumoral Immunomodulation. Methods Mol Biol 2058:133-154.

21. Vijayakumar G, Palese P, Goff PH. 2019. Oncolytic Newcastle disease virus expressing a checkpoint inhibitor as a radioenhancing agent for murine melanoma. EBioMedicine 49:96-105.

22. Schirrmacher V. 2016. Fifty Years of Clinical Application of Newcastle Disease Virus: Time to Celebrate! Biomedicines 4.

586 23. Zamarin D, Palese P. 2012. Oncolytic Newcastle disease virus for cancer therapy: old challenges and new directions. Future Microbiol 7:347-67.

24. Steel J, Burmakina SV, Thomas C, Spackman E, Garcia-Sastre A, Swayne DE, Palese P. 2008. A

591 25. Ma J, Lee J, Liu H, Mena I, Davis AS, Sunwoo SY, Lang Y, Duff M, Morozov I, Li Y, Yang J, combination in-ovo vaccine for avian influenza virus and Newcastle disease virus. Vaccine 26:522-31. Garcia-Sastre A, Richt JA, Ma W. 2017. Newcastle disease virus-based H5 influenza vaccine protects chickens from lethal challenge with a highly pathogenic H5N2 avian influenza virus. NPJ Vaccines 2:33. 
26. Grieves JL, Yin Z, Garcia-Sastre A, Mena I, Peeples ME, Risman HP, Federman H, Sandoval MJ, Durbin RK, Durbin JE. 2018. A viral-vectored RSV vaccine induces long-lived humoral

27. Liu RQ, Ge JY, Wang JL, Shao Y, Zhang HL, Wang JL, Wen ZY, Bu ZG. 2017. Newcastle disease virus-based MERS-CoV candidate vaccine elicits high-level and lasting neutralizing

29. Carnero E, Li W, Borderia AV, Moltedo B, Moran T, Garcia-Sastre A. 2009. Optimization of

30. Gao Q, Park MS, Palese P. 2008. Expression of transgenes from newcastle disease virus with a segmented genome. J Virol 82:2692-8.

31. DiNapoli JM, Kotelkin A, Yang L, Elankumaran S, Murphy BR, Samal SK, Collins PL, Bukreyev A. 2007. Newcastle disease virus, a host range-restricted virus, as a vaccine vector for intranasal immunization against emerging pathogens. Proc Natl Acad Sci U S A 104:9788-93.

32. Park MS, Steel J, Garcia-Sastre A, Swayne D, Palese P. 2006. Engineered viral vaccine constructs with dual specificity: avian influenza and Newcastle disease. Proc Natl Acad Sci U S

615 33. Nakaya T, Cros J, Park MS, Nakaya Y, Zheng H, Sagrera A, Villar E, Garcia-Sastre A, Palese P. 2001. Recombinant Newcastle disease virus as a vaccine vector. J Virol 75:11868-73.

617 34. Hsieh CL, Goldsmith JA, Schaub JM, DiVenere AM, Kuo HC, Javanmardi K, Le KC, Wrapp D, 618 Lee AG, Liu Y, Chou CW, Byrne PO, Hjorth CK, Johnson NV, Ludes-Meyers J, Nguyen AW, 619 Park J, Wang N, Amengor D, Maynard JA, Finkelstein IJ, McLellan JS. 2020. Structure-based 620 Design of Prefusion-stabilized SARS-CoV-2 Spikes. bioRxiv doi:10.1101/2020.05.30.125484.

621 35. Buchholz UJ, Finke S, Conzelmann KK. 1999. Generation of bovine respiratory syncytial virus WP, Lee B. 2017. Efficient and Robust Paramyxoviridae Reverse Genetics Systems. mSphere 2.

37. Hernandez R, Brown DT. 2010. Growth and maintenance of chick embryo fibroblasts (CEF). Curr Protoc Microbiol Appendix 4:4I. 
628 38. Ayllon J, Garcia-Sastre A, Martinez-Sobrido L. 2013. Rescue of recombinant Newcastle disease virus from cDNA. J Vis Exp doi:10.3791/50830.

630 39. Amanat F, White KM, Miorin L, Strohmeier S, McMahon M, Meade P, Liu WC, Albrecht RA, Simon V, Martinez-Sobrido L, Moran T, Garcia-Sastre A, Krammer F. 2020. An In Vitro Microneutralization Assay for SARS-CoV-2 Serology and Drug Screening. Curr Protoc

Acknowledgements

636 We thank Dr. Benhur Lee to kindly share the BSRT7 cells. We thank Dr. Thomas Moran for the 1C7C7 antibody. We thank Dr. Robert Coffman and Dynavax for providing the CpG 1018. We thank Dr. Andrew Pekosz from Johns Hopkins Bloomberg School of Public Health for providing the B.1.351 challenge virus. We thank Dr. Randy Albrecht for support with the BSL3 facility and procedures at the Icahn School of Medicine at Mount Sinai, New York. We also thank Nexelis for performing the pseudo-particle neutralization assay. This work was partially supported by an NIAID funded Center of Excellence for Influenza Research and Surveillance (CEIRS, HHSN272201400008C, P.P.) and a grant from an anonymous philanthropist to Mount Sinai (PP, FK, AG-S). This work was also supported, in part, by the Bill \& Melinda Gates Foundation [INV-021239]. Under the grant conditions of the foundation, a Creative Commons Attribution 4.0 generic License has already been assigned to the Author Accepted Manuscript version that might arise from this submission. The findings and conclusions contained within are those of the authors and do not necessarily reflect positions or policies of the Bill \& Melinda Gates Foundation. KS is supported by the Japanese Society for the Promotion of Science (JSPS) Overseas Research Fellowship.

\section{Author contributions}

653 Conceptualization and design: PP, FK, AG-S. and WS; construction and preparation of the vaccines: WS, 654 SM, YL, SS, IGD; GMP lots vaccine preparation: IVAC, GPO, Instituto Butantan, PATH; mouse 655 immunization and in vitro serological assays: WS, YL, IGD, KS ; mouse and hamster challenge and plaque 656 assays: WS, YL, IGD; micro-neutralization assay: FA; data analysis: PP, WS, YL, IDG, FA, AG-S, FK; 657 virus reagents: FA, LC, MS, IM, RR, SJ; first draft of the manuscript: PP and WS; manuscript review and 658 editing, all authors.

\section{Competing interests}


661 The Icahn School of Medicine at Mount Sinai has filed patent applications entitled "RECOMBINANT

662 NEWCASTLE DISEASE VIRUS EXPRESSING SARS-COV-2 SPIKE PROTEIN AND USES

663 THEREOF" which names PP, FK, WS and AG-S. as inventors. The AG-S laboratory has received research

664 support from Pfizer, Senhwa Biosciences, Kenall Manufacturing, Avimex, Johnson \& Johnson, Dynavax,

665 7Hills Pharma, Pharmamar, ImmunityBio, Accurius, Merck and Nanocomposix, and AG-S has consulting 666 agreements for the following companies involving cash and/or stock: Vivaldi Biosciences, Contrafect, 667 7Hills Pharma, Avimex, Vaxalto, Pagoda, Accurius, Esperovax, Farmak, Applied Biological Laboratories 668 and Pfizer.

672 Figure .1 Design of the NDV-HXP-S construct. (A) Structure and design of the NDV-HXP-S genome.

673 The ectodomain of the spike was connected to the transmembrane domain and cytoplasmic tail (TM/CT)

674 of the F protein. The original polybasic cleavage site was removed by mutating RRAR to A. The HexaPro 675 (F817P, A892P, A899P, A942P, K986P and V987P) stabilizing mutations were introduced. The sequence 676 was codon-optimized for mammalian host expression (B) Protein staining of NDV-HXP-S. WT NDV as 677 well as NDV-HXP-S were partially-purified from allantoic fluid through a sucrose cushion and 678 resuspended in PBS. Five $\mu \mathrm{g}$ (1) and ten $\mu \mathrm{g}$ (2) of the WT NDV, as well as ten $\mu \mathrm{g}$ of NDV-HXP-S were 679 resolved on 4-20\% SDS-PAGE. The viral proteins were visualized by Coomassie Blue staining (L, S, $680 \mathrm{HN}, \mathrm{N}, \mathrm{P}$ and $\mathrm{M})$.

Figure 2. Low doses of inactivated NDV-HXP-S induce protective antibody response in mice. (A)

683 Design of the study. Nine to ten-week old female BALB/c mice were used. Group 1 to 5 were vaccinated 684 with unadjuvanted NDV-HXP-S at $1 \mu \mathrm{g}, 0.3 \mu \mathrm{g}, 0.1 \mu \mathrm{g}, 0.03 \mu \mathrm{g}$ and $0.01 \mu \mathrm{g}$ per mouse, respectively. 685 Group 6 and 7 were vaccinated with $0.1 \mu \mathrm{g}$ of NDV-HXP-S with 10 or $30 \mu \mathrm{g}$ of CpG 1018 per mouse, 686 respectively. Group 8 and 9 were vaccinated with $0.03 \mu \mathrm{g}$ of NDV-HXP-S with 10 or $30 \mu \mathrm{g}$ of CpG 1018 687 per mouse, respectively. Group 10 was vaccinated with $1 \mu \mathrm{g}$ of WT NDV as the negative control. The 688 vaccine was administered via the intramuscular (I.M.) route at D0 and D21. Blood was collected at D21 689 and D43. Mice were sensitized with Ad5-hACE2 at D45 and challenged with $10^{5}$ PFU of the USA690 WA1/2020 strain. (B) Spike-specific serum IgG. Antibodies in post-prime (D21) and post-boost (D43) 691 sera were measured by ELISAs. Geometric mean titer (GMT) represented by area under the curve (AUC) 692 was graphed. (C) Neutralizing activity of serum antibodies. Post-boost sera from group 1, group 7 and 693 group 10 were pooled within each group and tested in neutralization assays against the USA-WA1/2020 694 strain (WT), the B.1.351 variant and B 1.1.7 variant in technical duplicate. Serum dilutions inhibiting 
$50 \%$ of the infection $\left(\mathrm{ID}_{50}\right)$ were plotted. (LoD: limit of detection; $\mathrm{LoD}=1: 20 ; \mathrm{An} \mathrm{ID}_{50}=1: 10$ was assigned to negative samples) (D) Viral load in the lungs. Lungs of a subset of animals $(n=4)$ from each group were collected at day 2 and day 5 post-challenge. The whole lungs were homogenized in $1 \mathrm{~mL}$ of PBS.

698 Viral titers were measured by plaque assay on Vero E6 cells and plotted as GMT of PFU/mL. (LoD=50

$699 \mathrm{PFU} / \mathrm{mL}$; A titer of $25 \mathrm{PFU} / \mathrm{mL}$ was assigned to negative samples). Statistical difference was analyzed by ordinary one-way ANOVA corrected for Dunnett's multiple comparisons test $(* * * *,<0.0001)$

Figure 3. Inactivated NDV-HXP-S induces protective antibody response in hamsters. (A) Design of the study. Eighteen to twenty- week old female Golden Syrian hamsters were used. Group 1 to 3 were vaccinated with $5 \mu \mathrm{g}$ of NDV-HXP-S without adjuvants, with CpG 1018 and AddaVax, respectively. Group 4 was vaccinated with $5 \mu \mathrm{g}$ of WT NDV as the negative control. Group 5 was not vaccinated. The vaccine was administered via the intramuscular (I.M.) route at D0 and D21. Blood was collected at D21 and D39. Group 1-4 were challenged with $10^{4}$ PFU of USA-WA1/2020 strain at D42. Group 5 was mockchallenged with PBS. (B) Spike-specific serum IgG. Antibodies in post-prime (D21) and post-boost (D39) sera were measured by ELISAs. GMT endpoint titer was graphed. (C) Neutralizing activity of serum antibodies. Post-boost sera from group 1-4 were pooled within each group and tested in neutralization assays against USA-WA/2020 strain (WT), B.1.351 variant and B 1.1.7 variant in technical

712 duplicate. Serum dilutions inhibiting $50 \%$ of the infection $\left(\mathrm{ID}_{50}\right)$ were plotted. $\left(\mathrm{LoD}=1: 50 ; \mathrm{An} \mathrm{ID}_{50}=1: 25\right.$

713 was assigned to negative samples) (D) Body weight change of hamsters. Body weights were recorded for

7145 days after challenge. (E) Viral load in the lungs and nasal washes. Lower right and upper right lung

715 lobes of a subset of animals ( $n=4$ for group 1-4; $n=3$ for group 5) from each group were collected at day 2

716 and day 5 post-challenge. Each lung lobe was homogenized in $1 \mathrm{~mL}$ PBS. Nasal washes were collected in $7170.4 \mathrm{~mL}$ of PBS. Viral titers were measured by plaque assay on Vero E6 cells and plotted as GMT of $718 \mathrm{PFU} / \mathrm{mL}$ (LoD=50 PFU/mL; A titer of $25 \mathrm{PFU} / \mathrm{mL}$ was assigned to negative samples). Statistical 719 difference was analyzed by two-way ANOVA corrected for Dunnett's multiple comparisons test (**, $720 \mathrm{p}<0.005 ; * * *, \mathrm{p}<0.0005 ; * * * *,<0.0001)$

Figure 4. GMP lots of inactivated NDV-HXP-S produced by influenza virus vaccine manufacturers 724 induce protective antibody response in hamsters. (A) Design of the study. Nine to eleven-week old 725 female Golden Syrian hamsters were used. Group 1 to 6 were vaccinated with $1 \mu \mathrm{g}$ of spike antigen of 726 inactivated NDV-HXP-S from GPO, IVAC and Butantan in the absence or presence of CpG 1018. Group 7277 was vaccinated with PBS as the negative control. Group 8 was not vaccinated (HC, healthy controls).

728 The vaccine was administered via the intramuscular (I.M.) route at D0 and D21. Blood was collected at 
D0, D21 and D33. Group 1-7 were challenged with $10^{4} \mathrm{PFU}$ of the USA-WA1/2020 strain at D35. Group

7308 was mock-challenged with PBS. (B) Spike-specific serum IgG. Antibodies in pre-vaccination (D0),

731 post-prime (D21) and post-boost (D33) sera were measured by ELISAs. GMT endpoint titers were

732 graphed. (C) Neutralizing activity of serum antibodies. Pseudo-particle neutralization assay was

733 performed by Nexelis to measure neutralization titers of post-boost sera (D33). Human convalescent sera

734 were included in the same assay as the controls $\left(\mathrm{LoD}=1: 25 ; \mathrm{An}^{\mathrm{ID}} \mathrm{D}_{50}=1: 12.5\right.$ was assigned to negative

735 samples). (D) Body weight changes of hamsters. Body weights were monitored for 5 days after challenge.

736 (E) Viral load in the lungs. Lower right and upper right Lung lobes of a subset of animals ( $\mathrm{n}=4)$ from

737 each group were collected at day 2 and day 5 post-challenge. Each lung lobe was homogenized in $1 \mathrm{~mL}$

738 PBS. (F) Viral load in nasal washes and nasal turbinates. At day 2 and day 5 post-challenge, nasal washes

739 were collected in $0.4 \mathrm{~mL}$ of PBS. Nasal turbinates were homogenized in $0.5 \mathrm{~mL}$ PBS. Viral titers were

740 measured by plaque assay on Vero E6 cells and plotted as GMT of PFU/mL (LoD=50 PFU/mL; A titer of

$74125 \mathrm{PFU} / \mathrm{mL}$ was assigned to negative samples). Statistical difference was analyzed by ordinary one-way

742 ANOVA corrected for Dunnett's multiple comparisons test $(* * * *, p<0.0001)$

Figure 5. Live NDV-HXP-S via the intranasal route induces protective antibody responses in used. Group 1 was vaccinated with $10^{6}$ EID $_{50}$ of live NDV-HXP-S. Group 2 was vaccinated with $10^{6}$ EID $_{50}$ of live WT NDV as the vector-only control. Group 3 was vaccinated with PBS as the negative control. Group 4 were the healthy controls. The vaccine was administered via the intranasal (I.N.) route at D0 and D22. Blood was collected at D22 and D41. Group 1-3 were challenged with $10^{5}$ PFU of the USAWA1/2020 strain at D44. Group 4 was mock-challenged with PBS. (B) Spike-specific serum IgG. Antibodies in post-prime (D22) and post-boost (D41) sera were measured by ELISAs. GMT endpoint titers were graphed. (C) Neutralizing activity of serum antibodies. Post-boost sera from group 1-3 were pooled within each group and tested in neutralization assay against USA-WA/2020 strain (WT), B.1.351 variant and B 1.1.7 variant in technical duplicate. Serum dilutions inhibiting $50 \%$ of the infection $\left(\mathrm{ID}_{50}\right)$ were plotted ( $\mathrm{LoD}=1: 50 ; \mathrm{An} \mathrm{ID}_{50}=1: 25$ was assigned to negative samples). (D) Body weight change of hamsters. Body weight was recorded for 5 days after challenge. (E) Viral load in the lungs and nasal

758 washes. Lower right and upper right lung lobes of a subset of animals $(n=3)$ from each group were 759 collected at day 2 and day 5 post-challenge. Each lung lobe was homogenized in $1 \mathrm{~mL}$ PBS. Nasal washes 760 were collected in $0.4 \mathrm{~mL}$ of PBS. Viral titers were measured by plaque assay on Vero E6 cells and plotted 761 as GMT of PFU/mL ( $\mathrm{LoD}=50 \mathrm{PFU} / \mathrm{mL}$; A titer of $25 \mathrm{PFU} / \mathrm{mL}$ was assigned to negative samples).

762 Statistical difference was analyzed by two-way ANOVA corrected for Dunnett's multiple comparisons 
test $(* *, p<0.005 ; * * *, p<0.0005)$

Figure 6. Intranasal prime followed by intramuscular boost of live NDV-HXP-S induces protective antibody responses in mice. (A) Design of the study. Seven to nine- week-old female BALB/c mice were used. Group 1-3 were vaccinated with $10^{4}, 10^{5}$ and $10^{6}$ EID $_{50}$ of live NDV-HXP-S, respectively. Group 4 was vaccinated with $10^{6}$ EID $_{50}$ of WT NDV. Group 5 was mock-vaccinated with PBS. The vaccine was administered via the intranasal (I.N.) route at D0 and intramuscular (I.M.) route at D21. Blood was collected at D21 and D43. Mice were sensitized with Ad5-hACE2 at D40 and challenged with $10^{5} \mathrm{PFU}$ of the USA-WA1/2020 strain at D45. (B) Spike-specific serum IgG. Antibodies in post-prime (D21) and post-boost (D43) sera were measured by ELISAs. GMT AUC was graphed. (C) Neutralizing activity of serum antibodies. Post-boost sera from group 1-4 were pooled within each group and tested in neutralization assay against USA-WA/2020 strain (WT), B.1.351 variant and B 1.1.7 variant in technical duplicate. Serum dilutions inhibiting $50 \%$ of the infection $\left(\mathrm{ID}_{50}\right)$ were plotted $\left(\mathrm{LoD}=1: 50 ; \mathrm{An} \mathrm{ID}_{50}=1: 25\right.$ was assigned to negative samples). (D) Viral load in the lungs. Lungs of a subset of animals ( $\mathrm{n}=5)$ from each group were collected at day 2 and day 5 post-challenge. The whole lungs were homogenized in $1 \mathrm{~mL}$ PBS. Viral titers were measured by plaque assay on Vero E6 cells and plotted as GMT of PFU/mL ( $\mathrm{LoD}=50 \mathrm{PFU} / \mathrm{mL}$; A titer of $25 \mathrm{PFU} / \mathrm{mL}$ was assigned to negative samples). Statistical difference was analyzed by ordinary one-way ANOVA corrected for Dunnett's multiple comparisons test (****, $<0.0001)$

Figure 7. Inactivated NDV-HXP-S induces protective antibody response against challenge of SARSCoV-2 variants of concern. Eight to ten- week-old female BALB/c mice were either vaccinated with 1 $\mu \mathrm{g}$ of inactivated NDV-HXP-S or WT NDV (negative control). Two immunizations were performed via the intramuscular route at D0 and D21. At D44, mice were treated with Ad5-hACE2. At D49, one third of mice from each group was challenged with USA-WA1/2020, B.1.351 or P.1 strain. At day 2, lungs were harvested and homogenized in $1 \mathrm{~mL}$ PBS. Viral titers were measured by plaque assay on Vero E6 cells and plotted as GMT of PFU/mL ( $\mathrm{LoD}=50 \mathrm{PFU} / \mathrm{mL}$; A titer of $25 \mathrm{PFU} / \mathrm{mL}$ was assigned to negative samples). Statistical difference was analyzed by one-tailed $t$ test. The $\mathrm{p}$ values are indicated.

794 IgG1. ELISAs were performed using either anti-mouse IgG2a or IgG1 secondary antibody to determine 795 subclasses of IgG induced by (A) inactivated NDV-HXP-S related to the mouse study described in Figure 7962 or (B) live NDV-HXP-S related to the mouse study described in Figure 6. GMT AUC was plotted. 


\section{Figure S2. Inactivated NDV-HXP-S reduces SARS-CoV-2 induced lung pathology in hamsters.}

799 Related to the hamster study in Figure 4, Left lung lobes of hamsters collected at day 5 post-challenge

800 were fixed in neutral buffered formalin and cut into $5 \mu \mathrm{m}$ sections and stained with hematoxylin and eosin

801 (H\&E). All sections were evaluated by a veterinary pathologist who was blinded to the vaccination

802 groups to score (A) Amount of lung affected; (B) Perivascular inflammation; (C) Alveolar inflammation

803 and necrosis/fibrin; (D) Type II pneumocytes hyperplasia/cytopathy and (E) Epithelial

804 degeneration/necrosis, bronchial/bronchiolar inflammation, intraluminal debris.

806 Figure S3. Live NDV-HXP-S induces spike-specific mucosal IgA. Nasal washes were collected from

807 mice that were vaccinated with $10^{6}$ EID $_{50}$ of NDV-HXP-S or WT NDV (negative control) intranasally 3

808 weeks later. Spike-specific IgA in the nasal washes was measured by ELISAs. 
A

WT NDV

\begin{tabular}{llllll}
\hline$N P$ & $P$ & & &
\end{tabular}

NDV-HXP-S

$\mathbf{N P} \mathbf{P}$ HexaPro Spike $\mathbf{M}$

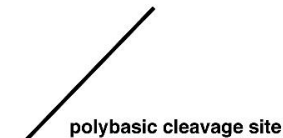

pasic cleavage site

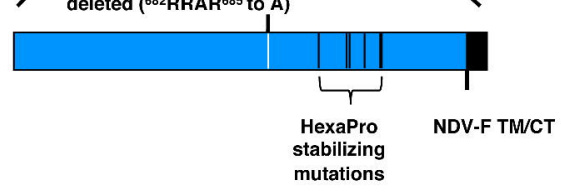

\section{HN}

L289A

L

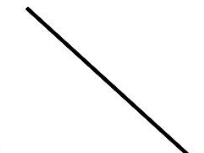

B

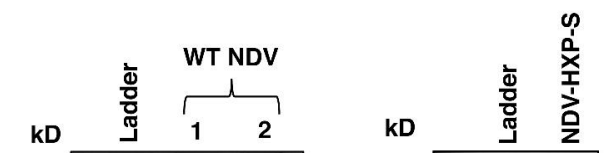

\section{Figure 1}




\begin{tabular}{|c|c|c|c|c|c|c|c|c|}
\hline \multirow[b]{2}{*}{$\begin{array}{l}\text { Group } \\
(n=8)\end{array}$} & \multicolumn{5}{|c|}{ Immunization } & \multirow{2}{*}{$\begin{array}{l}\text { Blood } \\
\text { draw } \\
\text { days }\end{array}$} & \multirow{2}{*}{$\begin{array}{l}\text { Ad5- } \\
\text { hACE2 }\end{array}$} & \multirow{2}{*}{$\begin{array}{l}\text { Challenge } \\
\text { (10 } 0^{5} \mathrm{PFU} \text { ) }\end{array}$} \\
\hline & $\begin{array}{c}\text { NDV-HXP-S } \\
\text { dose (ug) }\end{array}$ & $\begin{array}{c}\text { GpG } 1018 \\
\text { (ug) }\end{array}$ & Route & Prime & Boost & & & \\
\hline 1 & 1.0 & 0 & I.M. & DO & D21 & D21,43 & D45 & D50 \\
\hline 2 & 0.3 & 0 & I.M. & DO & D21 & D21,43 & D45 & D50 \\
\hline 3 & 0.1 & 0 & I.M. & DO & D21 & D21,43 & D45 & D50 \\
\hline 4 & 0.03 & 0 & I.M. & DO & D21 & D21,43 & D45 & D50 \\
\hline 5 & 0.01 & 0 & I.M. & DO & D21 & D21,43 & D45 & D50 \\
\hline 6 & 0.1 & 10 & I.M. & DO & D21 & $\mathrm{D} 21,43$ & D45 & D50 \\
\hline 7 & 0.1 & 30 & I.M. & DO & D21 & D21,43 & D45 & D50 \\
\hline 8 & 0.03 & 10 & I.M. & DO & D21 & D21,43 & D45 & D50 \\
\hline 9 & 0.03 & 30 & I.M. & DO & $\mathrm{D} 21$ & D21,43 & D45 & D50 \\
\hline 10 & WT NDV & 0 & I.M. & DO & D21 & D21,43 & D45 & D50 \\
\hline
\end{tabular}

B

Serum IgG

口 Post-prime

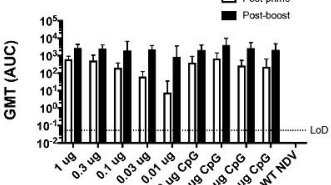

D

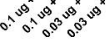

Day 2 lung titer

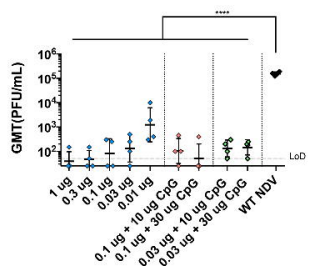

C Neutralization titers

(post-boost)

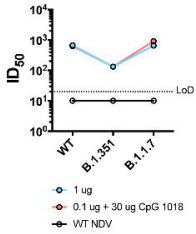

Day 5 lung titer

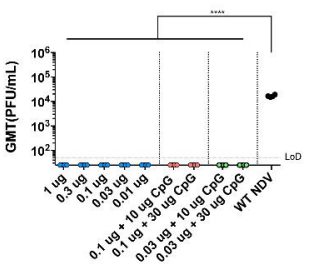




\begin{tabular}{|c|c|c|c|c|c|c|c|}
\hline \multirow[b]{2}{*}{ Group } & \multicolumn{5}{|c|}{ Immunization } & \multirow{2}{*}{$\begin{array}{c}\text { Blood } \\
\text { draw days }\end{array}$} & \multirow{2}{*}{$\begin{array}{l}\text { Challenge } \\
\text { (104 PFU) }\end{array}$} \\
\hline & $\begin{array}{l}\text { Vaccine } \\
\text { (5 ug total) }\end{array}$ & Adjuvant & Route & Prime & Boost & & \\
\hline $1(n=8)$ & NDV-HXP-S & No adjuvant & I.M. & Do & D21 & D21,39 & D42 \\
\hline $2(n=8)$ & NDV-HXP-S & $\mathrm{CpG} 1018$ & (1.M. & Do & D21 & D21,39 & D42 \\
\hline $3(n=8)$ & NDV-HXP-S & AddaVax & I.M. & Do & D21 & D21,39 & D42 \\
\hline $4(n=8)$ & WT NDV & None & I.M. & DO & D21 & D21,39 & D42 \\
\hline $5(\mathrm{n}=6)$ & Healthy Controls & N/A & I.M. & Do & D21 & D21,39 & Mock \\
\hline
\end{tabular}

B Serum IgG (ELISA)

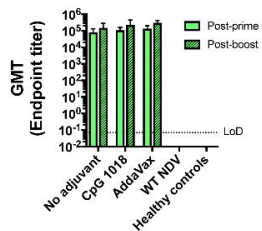

E Lower right lung lobe

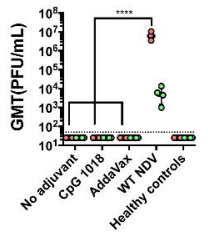

C Neutralization titers (post-boost)

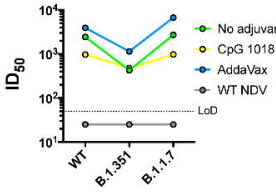

Upper right lung lobe

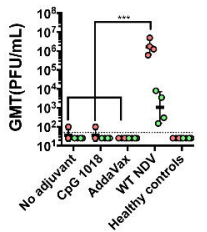

D SARS-CoV-2
(USA-WA1/2020)

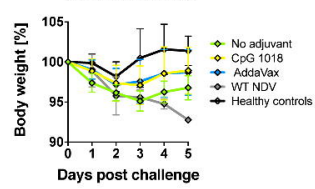

Nasal wash

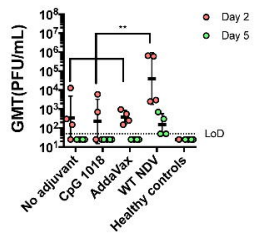


$\mathbf{A}\left(\mathrm{A}^{2} \quad \mathbf{D}\right.$

\begin{tabular}{|c|c|c|c|c|c|c|c|}
\hline \multirow[b]{2}{*}{$\begin{array}{c}\text { Group } \\
(n=8)\end{array}$} & \multicolumn{5}{|c|}{ Immunization } & \multirow{2}{*}{$\begin{array}{l}\text { Blood } \\
\text { draw } \\
\text { days }\end{array}$} & \multirow[b]{2}{*}{$\begin{array}{l}\text { Challonge } \\
\left.\text { (10 } 10^{4} \mathrm{PFU}\right)\end{array}$} \\
\hline & $\begin{array}{l}\text { Vaccines } \\
(1 \text { ug } \mathrm{S})\end{array}$ & $\begin{array}{c}\text { GpG } 1018 \\
(\mathrm{Ug})\end{array}$ & Route & Prime & Boost & & \\
\hline 1 & GPO & 0 & I.M. & Do & D21 & $D 0,21,33$ & D35 \\
\hline 2 & GPO & 100 & I.M. & Do & $\mathrm{D} 21$ & $\mathrm{D} 0,21,33$ & D35 \\
\hline 3 & IVAC & 0 & I.M. & Do & D21 & $\mathrm{D} 0,21,33$ & D35 \\
\hline 4 & IVAC & 100 & I.M. & Do & D21 & $\mathrm{DO}, 21,33$ & D35 \\
\hline 5 & Butantan & 0 & $I . M$ & Do & D21 & $\mathrm{D} 0,21,33$ & D35 \\
\hline 6 & Butantan & 100 & I.M. & Do & D21 & $\mathrm{D} 0,21,33$ & D35 \\
\hline 7 & PES & 0 & I.M. & Do & $\mathrm{D} 21$ & $\mathrm{D} 0,21,33$ & D35 \\
\hline 8 & $\mathrm{HC}$ & 0 & NA & Do & D21 & $\mathrm{D} 0,21,33$ & Mock \\
\hline
\end{tabular}

B Serum IgG (ELISA)

E

$\begin{array}{cc}\text { Day } 2 & \text { Day } 5 \\ \text { Lower right lung lobe } & \text { Lower right lung lobe }\end{array}$

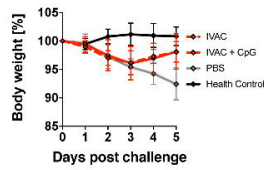

Butantan vaccine

Figure 4

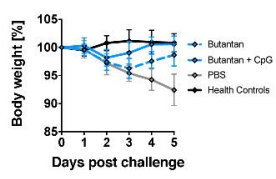

Day 2
Upper right lung lobe Upper right lung lobe

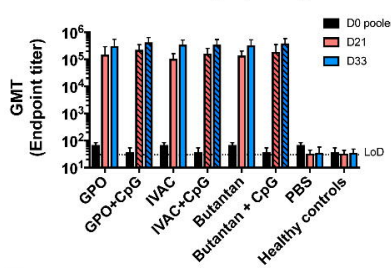

C SARS-Cov-2 Pseudoparticle
Neutralization Assay (D33 sera)

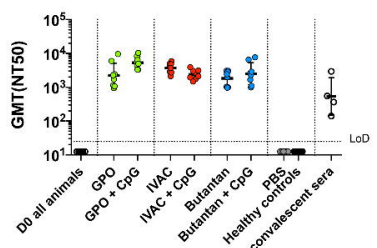

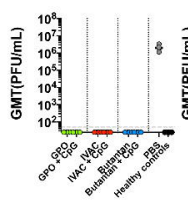

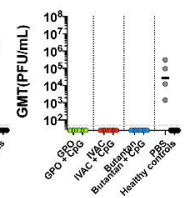

F

Day 2 Nasal wash

Day 5 Nasal wash
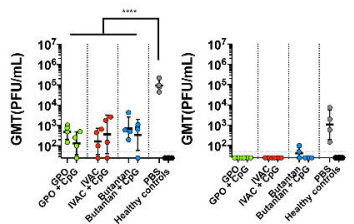

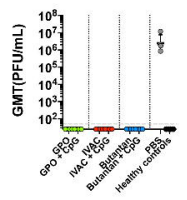

G
Day 2 Nasal turbinates
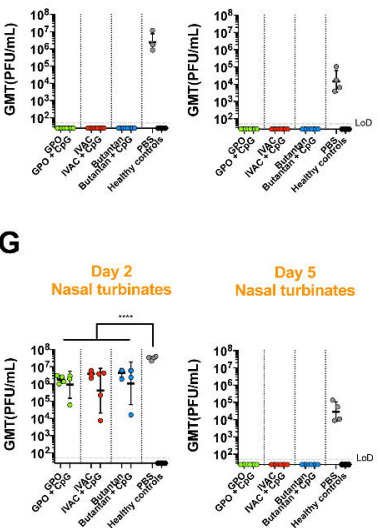

Day 5 Nasal turbinates

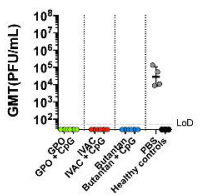


A

\begin{tabular}{|c|c|c|c|c|c|c|}
\hline \multirow{2}{*}{$\begin{array}{c}\text { Group } \\
(\mathrm{n}=6)\end{array}$} & Vaccine & Route & Prime & Boost & $\begin{array}{c}\text { Blood } \\
\text { Draw } \\
\text { days }\end{array}$ & $\begin{array}{c}\text { Challenge } \\
\left(10^{5} \text { PFU }\right)\end{array}$ \\
\cline { 2 - 5 } & NDV-HXP-S & I.N. & D0 & D22 & D22,41 & D44 \\
\hline 1 & WT NDV & I.N. & D0 & D22 & D22,41 & D44 \\
\hline 2 & PBS & I.N. & D0 & D22 & D22,41 & D44 \\
\hline 3 & Healthy controls & N/A & N/A & N/A & D22,41 & Mock \\
\hline 4 & & & & &
\end{tabular}

B

Serum IgG

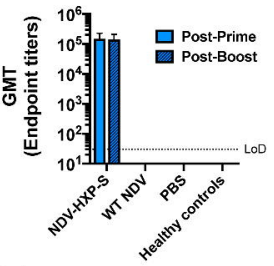

E

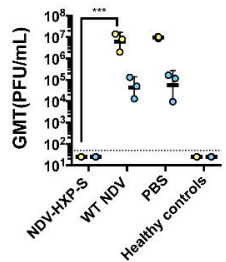

C

Neutralization titers (post-boost)

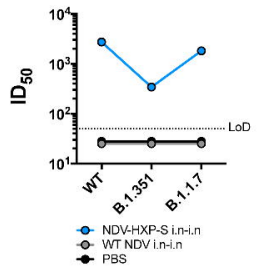

Upper right lung lobe

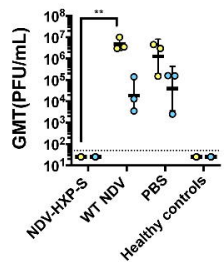

Nasal wash

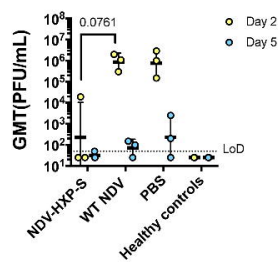

D SARS-CoV-2 (USA-WA1/2020)

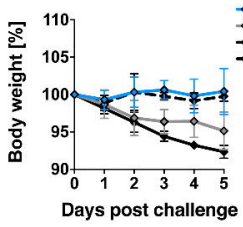

$\Leftrightarrow$ NDV-HXP-S

$\approx$ WT N

$\rightarrow$ Healthy controls

Figure 5 


\begin{tabular}{|c|c|c|c|c|c|c|}
\hline \multirow[b]{2}{*}{$\begin{array}{l}\text { Group } \\
(n=10)\end{array}$} & \multicolumn{3}{|c|}{ Immunization } & \multirow[b]{2}{*}{ Blood draw days } & \multirow[b]{2}{*}{$\begin{array}{l}\text { Ad5- } \\
\text { hACE2 }\end{array}$} & \multirow[b]{2}{*}{$\begin{array}{c}\text { Challenge } \\
\text { (10055 } \\
\text { PFU) }\end{array}$} \\
\hline & $\begin{array}{l}\text { NDV-HXP-S } \\
\text { dose } \\
\text { (EID50) }\end{array}$ & $\begin{array}{c}\text { Prime } \\
\text { (Intranasal) }\end{array}$ & $\begin{array}{c}\text { Boost } \\
\text { (intramuscular) }\end{array}$ & & & \\
\hline 1 & $10^{\wedge} 4$ & DO & D21 & D21,43 & D40 & D45 \\
\hline 2 & $10^{\wedge} 5$ & Do & D21 & D21,43 & D40 & D45 \\
\hline 3 & $10^{\wedge} 6$ & Do & D21 & D21,43 & D40 & D45 \\
\hline 4 & WT NDV & Do & D21 & D21,43 & D40 & D45 \\
\hline 5 & PBS & Do & D21 & D21,43 & D40 & D45 \\
\hline
\end{tabular}

\section{Figure 6}

B

Serum IgG

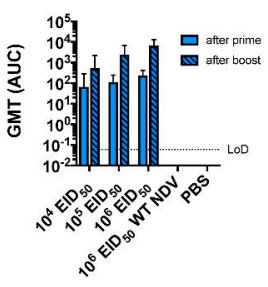

D

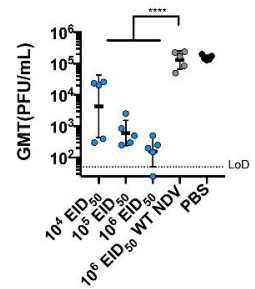

C

$$
\begin{aligned}
& \text { Neutralization titers } \\
& \text { (after boost) }
\end{aligned}
$$

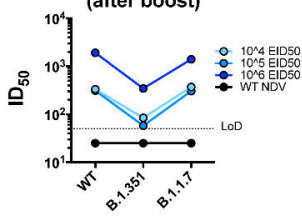

Day 5 lung titer

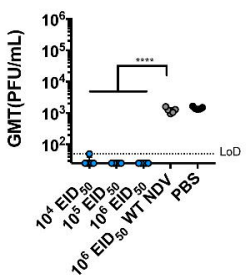




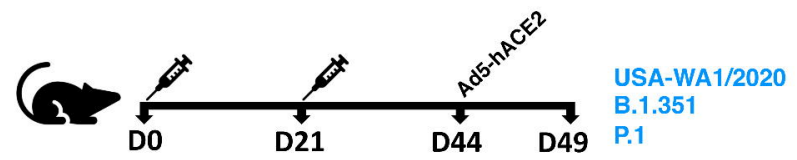

$\begin{array}{ccc}\text { WA1 } & \begin{array}{c}\text { B.1.351 } \\ \text { challenge }\end{array} & \begin{array}{c}\text { P.1 } \\ \text { challenge }\end{array}\end{array}$
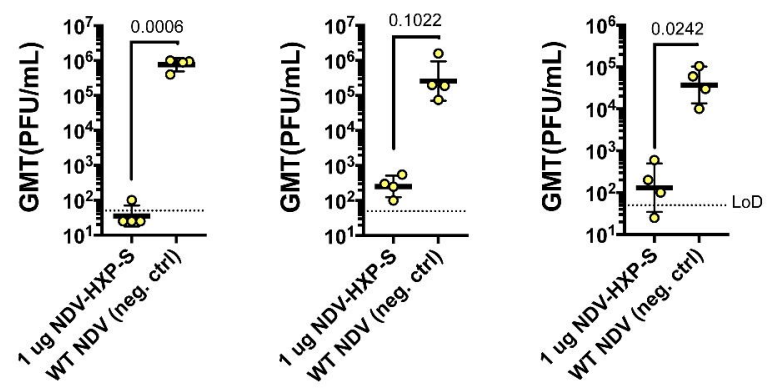
A

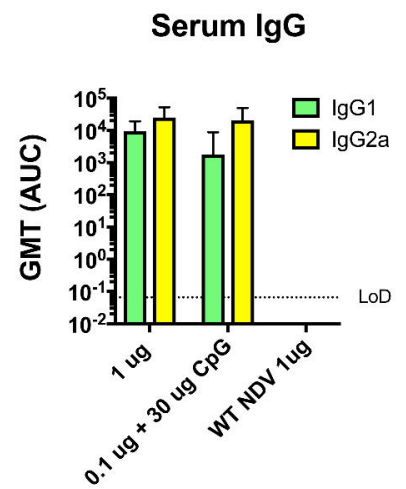

B

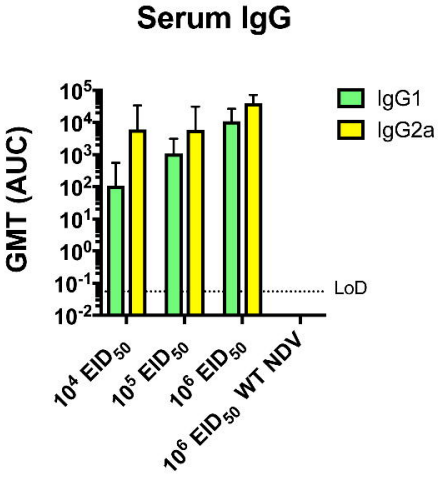


A Amount of lung affected

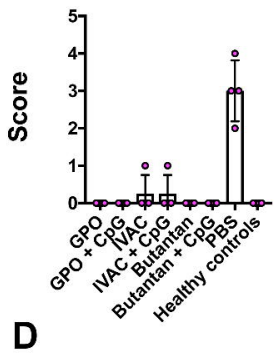

Type II pneumocyte hyperplasia/cytopathy

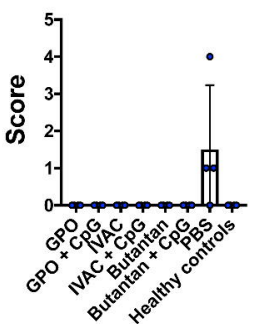

B

Perivascular inflammation

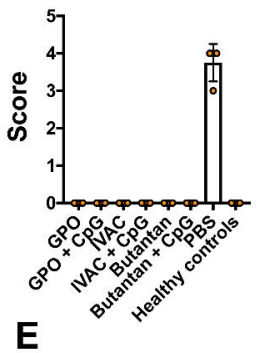

C

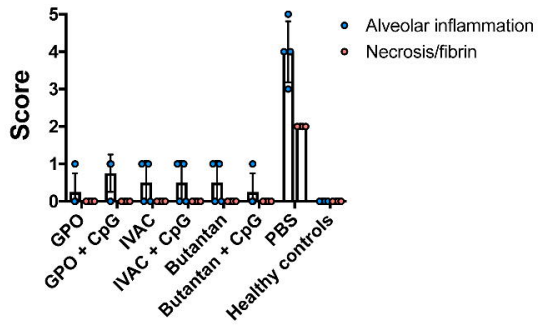

\section{Bronchi/Bronchioles}

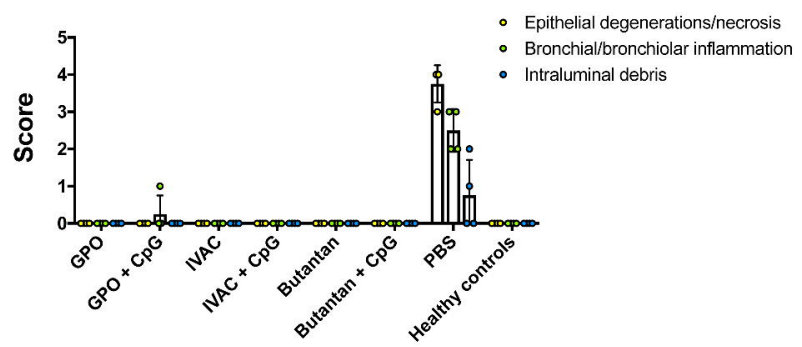


Nasal Wash IgA

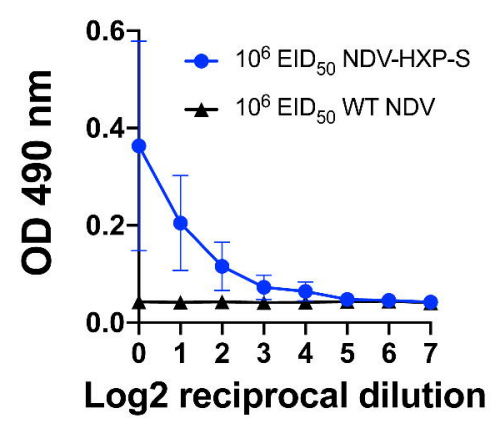

